\title{
Application of a Near Infrared Imaging System for Thermographic Imaging of the Space Shuttle during Hypersonic Re-Entry
}

\author{
Joseph N. Zalameda, Alan B. Tietjen", Thomas J. Horvath, Deborah M. Tomek \\ NASA Langley Research Center, Hampton VA 23681 \\ David M. Gibson, Jeff C. Taylor \\ Johns Hopkins University Applied Physics Laboratory, Laurel, MD 20723 \\ Steve Tack \\ Naval Air Warfare Center - Weapons Division, Pt. Mugu, CA 93042 \\ Brett C. Bush \\ Raytheon/Photon Research Associates, San Diego, CA 92121 \\ C. David Mercer \\ Aerospace Computing Inc., Hampton VA 23681 \\ Edward J. Shea \\ Futron Corporation, Hampton, VA 23666
}

\begin{abstract}
High resolution calibrated near infrared (NIR) imagery was obtained of the Space Shuttle's reentry during STS-119, STS-125, and STS-128 missions. The infrared imagery was collected using a US Navy NP-3D Orion aircraft using a long-range infrared optical package referred to as Cast Glance. The slant ranges between the Space Shuttle and Cast Glance were approximately 26-41 nautical miles at point of closest approach. The Hypersonic Thermodynamic Infrared Measurements (HYTHIRM) project was a NASA Langley led endeavor sponsored by the NASA Engineering Safety Center, the Space Shuttle Program Office and the NASA Aeronautics Research Mission Directorate to demonstrate a quantitative thermal imaging capability. HYTHIRM required several mission tools to acquire the imagery. These tools include pre-mission acquisition simulations of the Shuttle trajectory in relationship to the Cast Glance aircraft flight path, radiance modeling to predict the infrared response of the Shuttle, and post mission analysis tools to process the infrared imagery to quantitative temperature maps. The spatially resolved global thermal measurements made during the Shuttle's hypersonic reentry provides valuable flight data for reducing the uncertainty associated with present day ground-toflight extrapolation techniques and current state-of-the-art empirical boundary-layer transition or turbulent heating prediction methods. Laminar and turbulent flight data is considered critical for the development of turbulence models supporting NASA's next-generation spacecraft. This paper will provide the motivation and details behind the use of an upgraded NIR imaging system used onboard a Navy Cast Glance aircraft and describe the characterizations and procedures performed to obtain quantitative temperature maps. A brief description and assessment will be provided of the previously used analog NIR camera along with image examples from Shuttle missions STS-121, STS-115, and solar tower test. These thermal observations confirmed the challenge of a long-range acquisition during re-entry. These challenges are due to unknown atmospheric conditions, image saturation, vibration etc. This provides the motivation for the use of a digital NIR sensor. The characterizations performed on the digital NIR sensor included radiometric, spatial, and spectral measurements using blackbody radiation sources and known targets. An assessment of the collected data for three Space Shuttle atmospheric re-entries, STS-119, STS-125, and STS-128, are provided along with a description of various events of interest captured using the digital NIR imaging system such as RCS firings and boundary layer transitions. Lastly the process used to convert the raw image counts to quantitative temperatures is presented along with comparisons to the Space Shuttle's onboard thermocouples.
\end{abstract}

* ISTEF CSC, Kennedy Space Center, FL 32899

American Institute of Aeronautics and Astronautics

This material is declared a work of the U.S. Government and is not subject to copyright protection in the United States. 
AIAA 2010-245

\section{Nomenclature}

$\begin{array}{ll}\mathrm{i} & \text { counts } \\ \mathrm{k} & \text { counts threshold } \\ \mathrm{n} & \text { acquired image or frame number } \\ \mathrm{N} & \text { estimated Shuttle pixel resolution }\end{array}$

$\begin{array}{ll}\text { ABLT } & \begin{array}{l}\text { Asymmetric boundary layer transition } \\ \text { boundary layer transition } \\ \text { BLT }\end{array} \\ \text { CAPS } & \begin{array}{l}\text { computer aided pointing system } \\ \text { charge coupled device } \\ \text { CCD }\end{array} \\ \text { CFD } & \text { detailed test objective } \\ \text { DTO } & \text { flexible image transport system } \\ \text { FITS } & \text { Greenwich mean time } \\ \text { GMT } & \text { hypersonic thermodynamic infrared measurement } \\ \text { HYTHIRM } & \text { inter-range instrumentation group } \\ \text { IRIG } & \text { moderate resolution atmospheric transmission } \\ \text { MODTRAN } & \text { near infrared } \\ \text { NIR } & \text { nautical miles } \\ \text { NM } & \text { point of closest approach } \\ \text { PCA } & \text { reaction cured glass } \\ \text { RCG } & \text { reaction control system } \\ \text { RCS } & \text { signal to noise ratio } \\ \text { SNR } & \end{array}$

\section{Introduction}

This paper describes the motivation and details behind the use of a digital NIR imaging system used onboard a Navy Cast Glance aircraft and describe the characterizations and procedure performed to obtain quantitative temperature imagery of the Space Shuttle during hypersonic re-entry. The NASA Langley Research Center led Hypersonic THermodynamic InfraRed Measurement (HYTHIRM) project culminated in the acquisition of highresolution calibrated infrared imagery of the Space Shuttle during hypersonic atmospheric entry for STS-119, STS125 and STS-128 missions flown in 2009. The thermal imaging provided a unique and never before observed perspective on the global distribution of surface temperature and the state of the airflow (i.e., laminar/turbulent) over the entire windward surface of the Shuttle during hypersonic re-entry ${ }^{1}$. The thermal imagery represented several years of advocacy within the aerothermodynamics technical community, sponsorship by the NASA Engineering Safety Center, the Space Shuttle Program Office, the Hypersonic project within the NASA Aeronautics Research Mission Directorate, and careful planning and mission execution by a coalition of NASA, Navy, government labs, and contractor personnel. On Space Shuttle Discovery's March 2009 STS-119 mission, NASA flew a specially modified thermal protection system tile and instrumentation package to monitor heating effects from boundary layer transition (BLT) during re-entry ${ }^{2}$. BLT occurs when the smooth, laminar flow of air close to the Shuttle's surface is disturbed and becomes turbulent resulting in surface temperature increases. On STS-119, the windward airflow on the port wing was deliberately disrupted by a four-inch wide and quarter-inch tall "speed bump" built into the modified tile intended to promote transition to turbulence near Mach 15. In coordination with this flight experiment, the HYTHIRM team positioned a US Navy NP-3D Orion aircraft approximately 28 nautical miles below Discovery and remotely monitored surface temperature of the Shuttle at Mach 8.4 using a long-range infrared optical package referred to as Cast Glance ${ }^{3}$. The imagery from this mission not only captured the expected thermal footprint of the turbulent flow downstream of the wing protuberance, but a much larger area of turbulent flow on the opposing starboard wing that was not anticipated. The global thermal imagery obtained from the aircraft complemented the data collected with an onboard instrumentation package consisting of strategically placed thermocouples.

Approximately two months later, May 2009, the same Navy aircraft successfully monitored the surface temperatures of STS-125, Shuttle Atlantis not configured with a speed bump, traveling at approximately Mach 14.3 during 
its return from the successful Hubble repair mission. During September 2009, STS-128 Discovery outfitted with a slightly taller bump (0.35 inches tall) intended to promote transition to turbulence near Mach 18 , had a successful encounter with the P-3 at Mach 14.7. Collectively, the spatially resolved global thermal measurements made during the Shuttle's hypersonic re-entry were intended to demonstrate the capability to collect scientific quality imagery in a reliable manner using available technology ${ }^{1}$. It is the intent of the project to analyze the imagery and provide the technical community critical flight data for reducing the uncertainty associated with present day ground-to-flight extrapolation techniques and current state-of-the-art empirical BLT or turbulent heating prediction methods. Laminar and turbulent flight data is considered critical for the validation of physics-based prediction methods, to stimulate the validation of laminar numerical models and the development of turbulence models supporting NASA's next-generation spacecraft under the Constellation program.

This paper will provide the motivation and details behind the use of a digital NIR imaging system and describe the characterizations and procedure performed to obtain quantitative temperature imagery. Past attempts by the Cast Glance aircraft to image the Space Shuttle during STS-121 and STS-115 missions has resulted in successful image captures, however the quality of the acquired data allowed for limited data processing ${ }^{4,5,6,7}$. During both missions, the Shuttle imagery was obtained using an analog based NIR imaging system, however due to image saturation, limited quantitative analysis could be performed. As a result, the analog NIR imaging system onboard the Cast Glance aircraft was characterized during a series of solar tower experiments. The solar tower tests were conducted at the Sandia National Laboratories' National Solar Thermal Test Facility in Albuquerque, New Mexico and consisted of imaging an 8x8 array of Shuttle tiles with Reaction Cured Glass (RCG) coatings. The tile array was instrumented with thermocouples. A series of solar reflectance mirrors were used to heat the array up to $2,000^{\circ} \mathrm{F}$ and flight NIR data from Cast Glance were obtained. As a result of these tests, the recommendation was made to enhance the ana$\log$ NIR imaging system with a digital NIR imaging camera and characterize the new camera. The characterizations performed on the digital NIR system included radiometric, spatial, and spectral measurements using blackbody radiation sources and known targets. The radiometric characterization was performed using calibrated blackbodies set at various temperatures. This characterization was used as input to a radiance model ${ }^{8}$ to predict the imaged NIR radiometric response of the Shuttle and allow for proper selection of the digital camera integration time to minimize pixel saturation. The spatial characterization was performed during flight conditions on selected stars. This technique allows for an estimation of the spatial response of the Cast Glance imaging system. The spectral characterization involved using a series of long pass filters with known spectral responses. Implementation of the digital NIR camera system has resulted in NIR imagery with improved dynamic range, instantaneous field of view, and signal to noise. An assessment of the collected data, using the digital NIR imaging sensor, for STS-119, STS125 , and STS-128, is provided. This assessment of the quality of the thermal imagery includes total number of images acquired, number of saturated pixels, integration time adjustments vs. image number, and estimated pixel resolution. In addition, imagery of interest captured using the NIR imaging system such as RCS firings and BLT are presented. The process used to convert the raw image counts to quantitative 2-dimensional temperature images using Moderate Resolution Atmospheric Transmission (MODTRAN) ${ }^{9}$ corrections is presented. A more rigorous analysis of the thermal imagery including 3-dimensional mapping is discussed in Ref. 8. Lastly, the HYTHIRM remotely measured temperature values are compared to the Space Shuttle's onboard thermocouples.

\section{Description and Imaging Results of Analog NIR Imaging System}

A picture of the Cast Glance aircraft, NIR optical tracking system, and analog NIR camera is shown in Fig. 1. Additional details about the Cast Glance aircraft can be found in Ref. 3. The analog NIR camera is the Cohu model 2672 with a charge coupled device (CCD) sensor of pixel array size of 752 x 582. The camera signal to noise value is approximately $55 \mathrm{~dB}$. A long pass filter with cut on at $0.750 \mu \mathrm{m}$ is used to remove the spectral energy in the visible band. The imaging optics utilizes a 7-inch aperture reflecting telescope viewing through the forward starboard visible/NIR window. The optics are vibration isolated and gyroscope stabilized to minimize image jitter, improve pointing accuracy, and allows for manual tracking (preferred method). The NIR imaging system spectral response is $0.850-1.1 \mu \mathrm{m}$. Shown in Fig. 2 is the analog camera system layout. To minimize potential saturation during a mission, the integration time can be changed in discrete steps of $0.008,0.004,0.002,0.001,0.0005,0.00025$, and 0.0001 seconds using a manually adjusted dial. The RS 170 analog video is connected to a Computer Aided Pointing System (CAPS) for inserting aircraft Global Positioning System information, altitude, gimbal pointing angle, and ground speed. The analog video is then connected to a video distribution amplifier where the video is split to an object tracking monitor and video time inserter. After the images are time stamped, the analog video is recorded on a mini-DVCAM ${ }^{\mathrm{TM}}$ tape. The dynamic range of the recorded images is 8 bits and the frame rate is $30 \mathrm{~Hz}$. Past attempts by the Cast Glance aircraft to image the Space Shuttle during STS-121 (July 2006) and STS-115 (September 


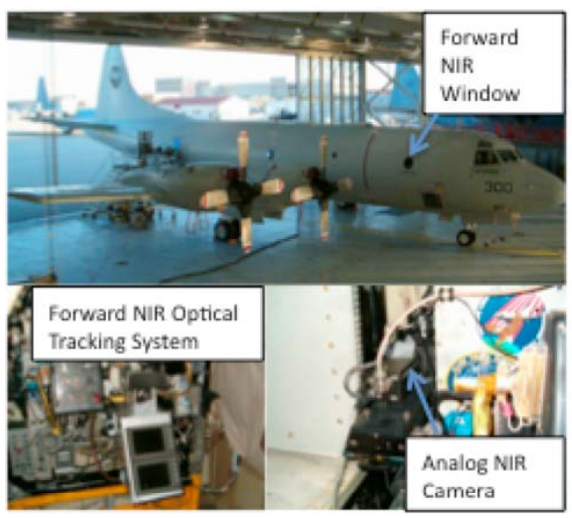

Fig. 1. Cast Glance analog NIR imaging system.

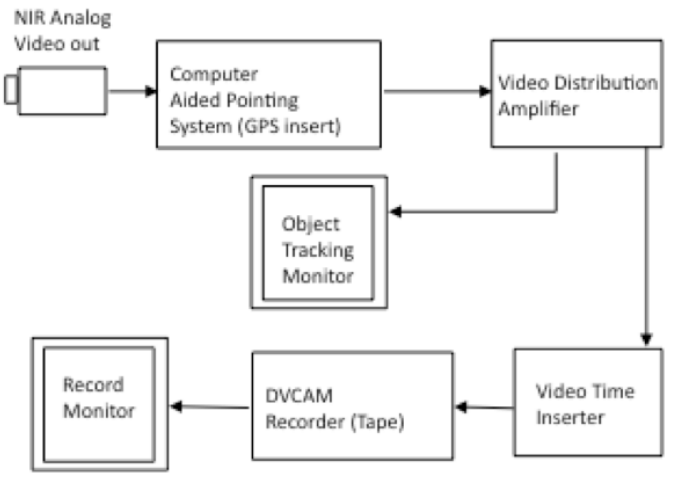

Fig. 2. Cast Glance analog NIR video signal layout.

2006) missions resulted in some success using the NIR analog system. During both missions, the Shuttle imagery was obtained, however due to image saturation limited quantitative analysis could be performed.

Example images are obtained by converting the mini-DVCAM video information into uncompressed digital files. Shown in Fig. 3 is the raw intensity image from STS-121 captured by Cast Glance aircraft at the approximate point of closest approach (PCA). The threshold image (saturated pixels highlighted in red) indicates significant image saturation over most of the windward surface of the vehicle. A partial histogram plot is also shown in Fig 3 indicating a significant number of saturated pixels. Shown in Fig. 4 is the raw intensity image from STS-115 captured by Cast Glance aircraft at approximate PCA. The threshold image shows saturated pixels at the Shuttle nose,
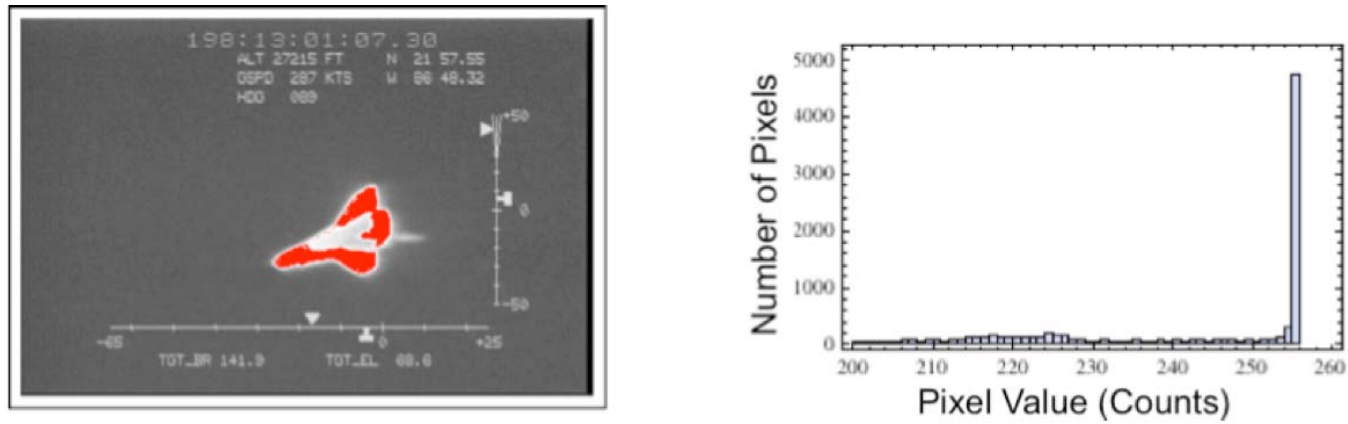

Fig. 3. Cast Glance raw NIR intensity image from STS-121 during re-entry with saturated pixels (red) and corresponding image histogram.
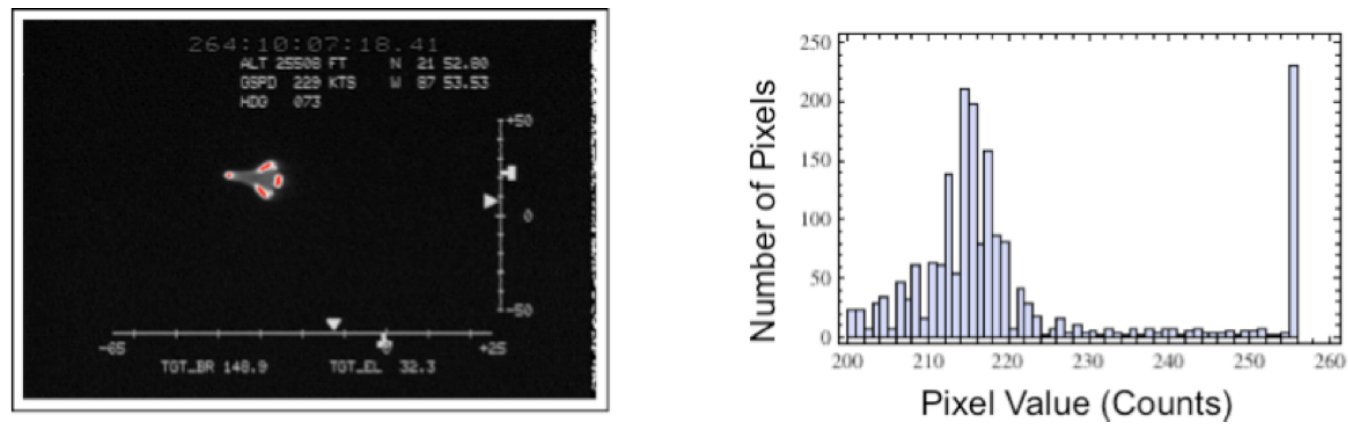

Fig. 4. Cast Glance raw NIR intensity image from STS-115 during re-entry with saturated pixels (red) and corresponding image histogram. 
wing leading edges, and body flap. A partial histogram plot is also shown in Fig 4 indicating the number of saturated pixels. Although the number of saturated pixels is small compared to STS-121 data, the slant range or optical path length is longer thus lowering the total number of pixels on target. This lower resolution image emphasizes the importance of each unsaturated pixel. A data collect priority is to minimize the number of saturated pixels during data acquisition. This can be challenging due to unknown atmospheric effects over a long optical path length that is changing with the approaching Shuttle target.

As a result the analog NIR imaging system onboard the Cast Glance aircraft was characterized during a series of solar tower experiments. The solar tower tests were conducted at the Sandia National Laboratories' National Solar Thermal Test Facility in Albuquerque, New Mexico ${ }^{10}$. Shown in Fig. 5 is the series of mirrors used to reflect the solar energy onto a tile array mounted on top of the tower. The testing consisted of imaging an $8 \times 8$ array of Space Shuttle RCG coated tiles during and after the application of solar energy. The $8 \times 8$ tile array (1.2 meter $x 1.2$ meter in size) was instrumented with thermocouples. A visible image of the tile array, partially illuminated, is shown in Fig. 6. A series of solar reflectance mirrors were used to heat the array up to $2,000^{\circ} \mathrm{F}$ and flight NIR data from Cast Glance was obtained. An example NIR image is shown in Fig. 7 and was acquired at a slant range of approximately $5 \mathrm{NM}$ and shows the tile array partially illuminated during cool down. For a given camera integration time, multiple frames during the cooling process were used to produce a calibration curve by using the known thermocouple values. Before using the images for analysis, a spatial stabilization algorithm based on object tracking through image correlation, was used to remove jitter. This allows for determining the area mean counts over the tile array as a

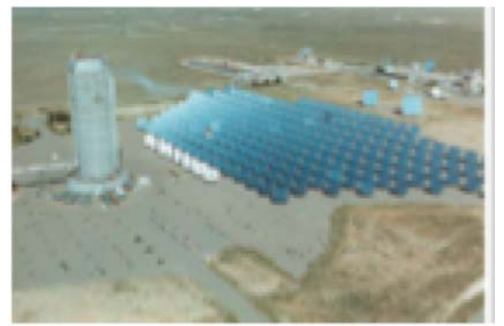

Fig. 5. Solar tower testing facility.

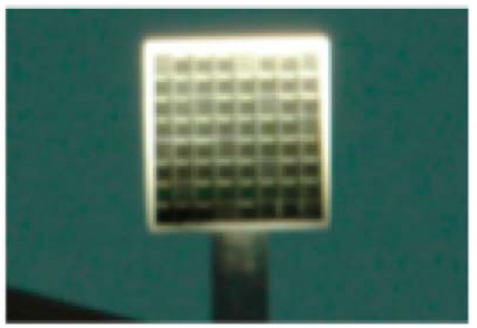

Fig. 6. Visible image of tile array.

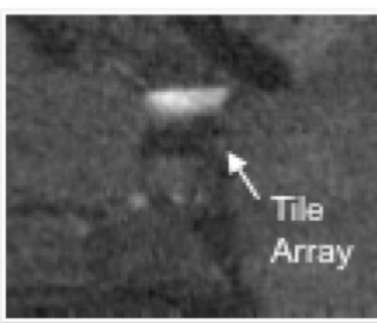

Fig. 7. Cast Glance NIR image.

function of time. The tile array radiant exitance was determined by averaging the thermocouple temperature values for a given area of interest using a known tile emissivity value. For a given surface area, the radiant exitance was converted to the in-band radiance ${ }^{11}$. The radiant energy or radiance was corrected for atmospheric path transmission, solar scattering from aerosols, and path radiance to produce the in-band radiance at the sensor aperture. These values were compared to the area-mean counts to produce a calibration curve. The calibration curve was then used to convert the image counts to object radiance for a given integration time taking into account the MODTRAN atmospheric modeling. The object radiance was converted to temperature by using Planck's black body radiation law $^{12}$ and known tile emissivity. Using this technique, a temperature image of the tile array can be calculated as shown in Fig. 8. The conversion of temperature using the analog NIR imaging system radiometric uncertainty is also shown in Table 1. As expected the lower the temperature the larger the 2 sigma errors due to the limitation of

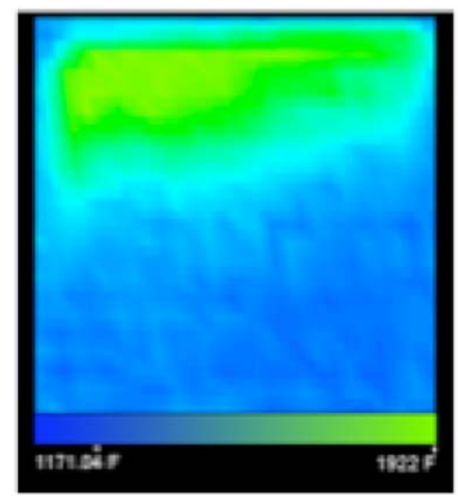

Table 1. Noise performance of analog camera.

\begin{tabular}{|l|l|}
\hline $\begin{array}{l}\text { Measured Temperature } \\
\text { Degrees F }\end{array}$ & $\begin{array}{l}2^{*} \text { Standard Deviation } \\
\text { Temperature Error, } \\
\text { +/-Degrees F }\end{array}$ \\
\hline 1126.0 & 450.0 \\
\hline 1302.4 & 212.4 \\
\hline 1522.0 & 72.0 \\
\hline 1615.6 & 46.8 \\
\hline 1974.2 & $\begin{array}{l}\text { Saturation (155 } \\
\text { counts) }\end{array}$ \\
\hline
\end{tabular}

Fig. 8 .Temperature image of tile array. 
the CCD sensor sensitivity at longer wavelengths. For the temperature range of interest, typical of a Shuttle reentry, the averaged 2-sigma errors (from Table 1) are greater than $+/-190^{\circ} \mathrm{F}$. These error values include both sensor noise and tile-to-tile emissivity non-uniformity. In addition it was noted the sensor saturation level was at 155 counts for the solar tower test data. This is much lower than the expected 8 bit (256 counts) dynamic range. This reduced dynamic range could have been the result of an incorrect gain or offset level in the camera electronics. As a result of these tests, showing poor signal to noise and limited dynamic range, the recommendation was made to replace the analog NIR sensor with a digital NIR imaging sensor and maintain the analog camera as a backup data acquisition system.

\section{Description and Characterization of Digital NIR Imaging System}

The digital NIR camera chosen to replace the analog camera is the Prosilica GC1380H. The camera pixel array size is $1360 \times 1024$ with a frame rate of $30 \mathrm{~Hz}$ at full resolution. The pixel array size is approximately a factor of 3 larger than the analog camera, which allows for an improved instantaneous field of view. The camera's dynamic range is 12 bits, which is a factor of 16 better than the analog camera. This improved dynamic range will help to minimize image saturation and or clipping. The integration time can be varied from $10 \mu \mathrm{sec}$. to $60 \mathrm{sec}$. and is continuously variable. This feature is a great improvement over the analog camera stepped dial knob adjustment. The camera has a GigE digital output. A laptop based digital acquisition system was developed to acquire the data, Fig 9a. The digital output improves signal to noise performance by reducing the cable noise associated with the analog system. The quoted camera signal to noise value is approximately $66 \mathrm{~dB}$ about 4 times better that the analog NIR camera. The spectral response of the camera's CCD sensor is $0.4-1.1 \mu \mathrm{m}$. A long pass filter with cut on at 0.850 $\mu \mathrm{m}$ is used to remove the spectral energy in the visible band. A picture of the digital camera, installed on the aircraft, is shown in Fig. 9b. The camera is half the size and weight. Size and weight are important considerations when upgrading flight hardware. Shown, Fig. 9c, is the digital acquisition system block diagram. The acquired digital data file is stored in the flexible image transport system (FITS) format ${ }^{13}$. Frame acquisition time is stamped with an inter-range instrumentation group (IRIG) time code provided by Cast Glance's GPS timing system

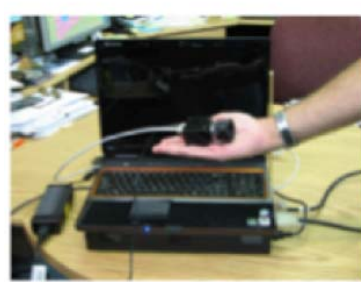

(a)

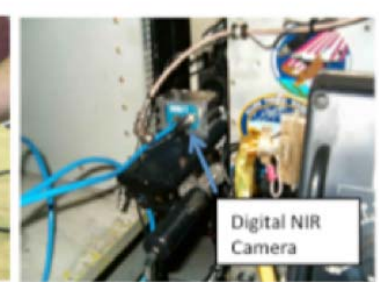

(b)

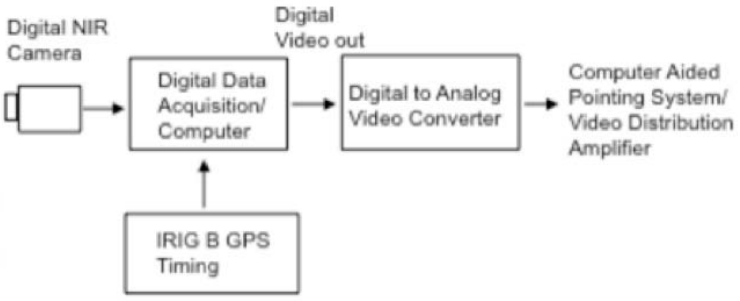

(c)

Fig. 9. Digital data acquisition system and NIR camera installed onboard Cast Glance aircraft with system layout diagram.

and the value is stored in the frame header. Custom data acquisition software was developed to control the camera's integration time in steps down to $10 \mu \mathrm{sec}$. The integration time can be adjusted in real time using the computer's keypad in fine, medium, or coarse steps. In addition, the integration time can be incremented to predefined values using the number keypad. The acquired digital video is displayed in real time on the computer's display and saturated pixels are colored in red. The characterizations performed on the digital NIR system included radiometric, spatial, and spectral measurements using blackbody radiation sources and known targets. These characterizations were used as input to the radiance $\operatorname{model}^{8}$ to predict the imaged NIR radiometric response of the Shuttle and allow for proper selection of the digital camera integration time to minimize pixel saturation.

\section{A. Radiometric}

The radiometric characterization was performed pre-flight using calibrated blackbody radiation sources set at various temperatures. The process involves the calibration of the measured radiance counts to actual temperature values. The calibration was performed in a temperature range of interest (up to $1,900^{\circ} \mathrm{F}$ ) at specified sensor integration times. The results are calibration curves that can then be used to convert the measured pixel intensity to quantitative radiance values. A series of spot check calibrations were performed during each of the three, STS-119, STS-125, and STS 128, data acquisition mission. This involved parking the Cast Glance aircraft at a known distance from the blackbody sources which is shown in Fig. 10a. The NIR imaging system tracking mirror is pointed at 


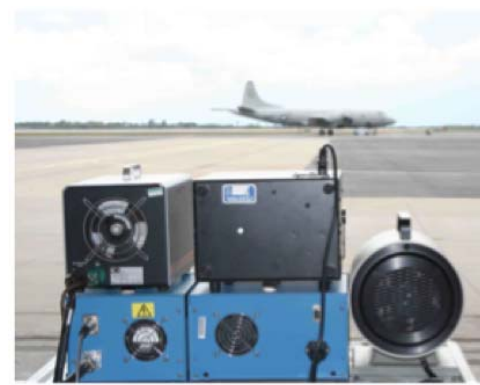

(a)

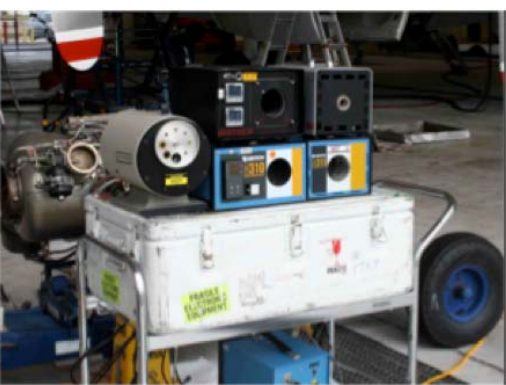

(b)

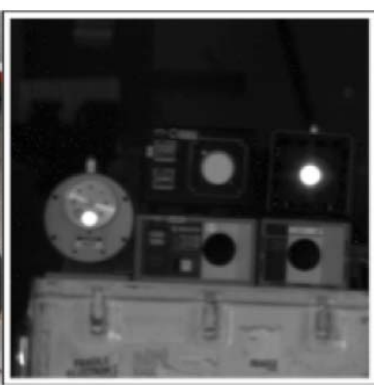

(c)

Fig. 10. Calibration setup using multiple blackbody radiation sources with example NIR image.

the blackbody sources, Fig. 10b, through the aircraft window, to observe the blackbody radiation. The NIR camera is focused on the blackbodies to acquire "spot check" data. This technique does not allow for full illumination of the camera's focal plane array, but is sufficient for comparison to existing calibration curves ${ }^{14}$. An example NIR image is shown in Fig. 10c. The spot check calibration data is then compared, mission-to-mission. This insures NIR imaging system is operating normally and the camera's radiometric response is consistent. The integration time was varied from 0.095 to 0.005 seconds for each measurement and at each integration time, 50 - 100 frames were acquired. In addition dark current measurements were made using a lens cap. The counts versus the in-band radiance at the aperture are determined given a known blackbody temperature. The radiance versus instrument response (counts per second) is typically a linear curve. These curves show excellent linearity (average regression squared value $=0.989$ ) and are documented in Ref. 14 along with the noise performance of the camera versus temperature. Preliminary noise values for the digital system, from calibration data, are less than $+/-10^{\circ} \mathrm{F}$ one sigma from $1350-$ $2200^{\circ} \mathrm{F}$, or about $+/-1.8 \%$ three sigma. This is an improvement over the NIR analog camera. Noise values below $1350{ }^{\circ} \mathrm{F}$ vary significantly with sensor integration time, but generally increase as the object's temperature decreases due to the rapid fall off of the black body curve within the spectral band of the sensor.

\section{B. Spatial}

The spatial characterization was performed during flight conditions on selected stars. This technique allows for an estimation of the spatial response of the Cast Glance digital NIR camera imaging system. The spatial point

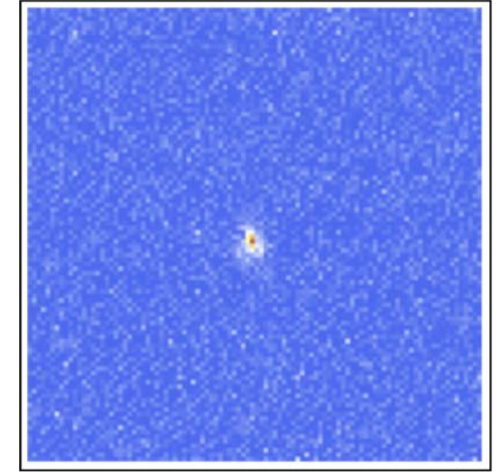

Fig. 11. Star calibration NIR image. spread response (blurring) is modeled as a Gaussian function and can be affected by the optics performance, flight turbulence, and jitter from aircraft vibrations. Multiple predetermined stars at varying Zenith angles were observed using the digital NIR imaging systems during a flight mission. A list of observable stars was finalized, crosschecked and programmed onto the Cast Glance's star data base CAPS and the CAPS reference numbers were identified for a sunset data acquisition flight mission. During the mission, flight calibration data were obtained on several stars and 2 planets (Venus and Saturn). The digital NIR camera was configured with a $750 \mathrm{~nm}$ cut on filter. Analysis of these star sequences yields the overall system's spatial resolution as flown for a given integration time. An example NIR observation (March 2009) of the star Sirius is shown in Fig. 11. The image size is a $100 \times 100$ pixel sub-window. The integration time was varied to determine Gaussian point spread function (PSF) as a function of integration time and was applied to the radiance modeling results ${ }^{8}$. This technique was only able to determine the PSF qualitatively. A more sophisticated laser interferometer based technique is recommended to more accurately determine the PSF. 


\section{Spectral}

The spectral characterization involved using a series of long pass filters with known spectral responses. This allows an estimate of the spectral response of the Cast Glance NIR imaging system using the digital NIR camera. For a given blackbody temperature, the spectral response of the imaging system can then be measured using this technique and will allow for more accurate temperature measurements. Spectral calibrations were performed on the digital NIR camera using 700, 750, 800, 850, 900, 950, 1,000 nanometer long pass NIR filters. The blackbody temperature was set at $1,600^{\circ} \mathrm{F}$. The integration time was varied from 0.08 to 0.000001 seconds for each measurement and at each integration time 50 - 100 frames were acquired. Data were acquired without a filter (open) and for each filter. The spectral response of the imaging system can then be determined using this technique. A drawback of this technique is that the response is estimated in steps of 50 nanometers. The planned future use of a spectrometer will allow for a more accurate determination of imaging system's spectral response function and provide greater spectral resolution. Therefore, the spectral response filter data was not used during the conversion to temperature.

\section{Assessment of STS-119, STS-125, and STS-128 Collected Data}

An assessment of the collected data, using the digital NIR imaging sensor, for STS-119, STS-125, and STS128, are provided. Implementation of the digital NIR camera system has resulted in NIR imagery with improved dynamic range, minimal saturation, and greater signal to noise. This assessment of the quality of the data collected includes number of saturated pixels, signal to noise performance, integration time adjustments vs. image number, and maximum number of pixels on target. In addition imagery of interest, captured using the NIR imaging system, such as reaction control system (RCS) firings, asymmetric boundary layer transition (ABLT), and detailed test objective (DTO) BLT are presented.

\section{A. STS-119 Mission}

The US Navy NP-3D Orion Cast Glance aircraft, using the digital NIR imaging system, captured STS-119 Shuttle Discovery, OV-103, mission (March 28, 2009) during re-entry at a PCA slant range of approximately 26.2 NM at GMT = 19:02:00, Fig.12. The total image acquisition time was over 9 minutes, the total number of acquired frames $\mathrm{n}=10,766$ and the integration time was varied from $80 \mathrm{~ms}$ down to $10 \mathrm{~ms}$, Fig. 13. The NIR acquisition file size was over 21 Gigabytes. Typically the maximum resolution is obtained at PCA. At PCA the slant range between the Shuttle and Cast Glance was 26.2 NM with corresponding image number $=3885$, the pixel resolution is not at a maximum, but is greatest at image number 3664. This is because the Shuttle is turning away from the aircraft at PCA. This was determined using an image histogram analysis method. The image histogram analysis method was used to analyze the NIR data frame by frame around PCA to determine the number of pixels on target and to determine the number of saturated pixels. The number of pixels on target is determined by summing the pixels with counts above a threshold for each acquired frame. The equation is given as:

$$
N=\sum_{i=k}^{4095} \text { Bincounts }_{i}
$$

where $i$ is the counts, $k$ is the counts threshold value, and $N$ is the estimated Shuttle pixel resolution. Because the

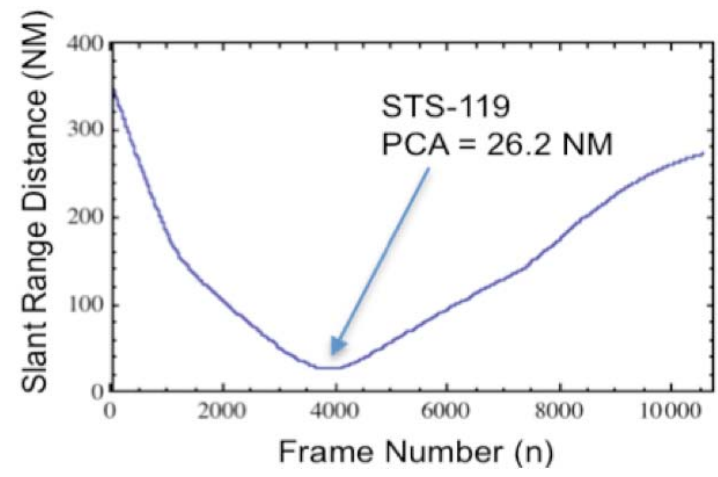

Fig. 12. STS-119 Cast Glance and Shuttle distance.

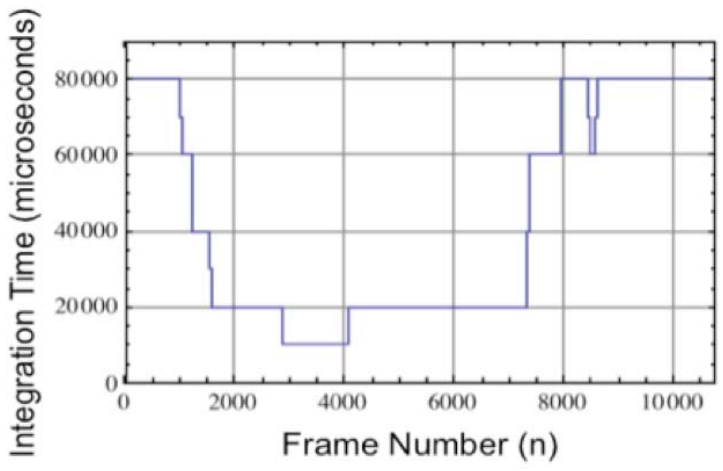

Fig. 13. NIR camera integration times. 
sky background is cool, compared to the Shuttle which is hot (higher pixel counts), the number of pixels associated with the Shuttle's windward surface can be estimated by summing all the pixels associated with a value greater than 250 counts $(k=250)$. This removes most pixel values associated with the background. This is plotted in Fig. 14 around the PCA frame numbers. This method is effective if the integration time is constant. Work is proceeding to modify this method to take into account integration time changes. The image histogram analysis technique is, nevertheless, important to determine which image or frame provided maximum pixels on target based on a combination of PCA and optimal viewing angle (closest to normal incidence to the Shuttle's windward surface). For STS-119, frame number $=3664$ provided the best pixel resolution, $N=6,081$, with a corresponding slant range distance of 28.2 NM at GMT = 19:01:53, seven seconds before PCA. At this point the Shuttle was traveling at approximately Mach 8.4.

Fig. 15 shows that no frames were saturated during the STS-119 data collection at PCA. This is due to the ability to adjust the integration time manually, in fine steps, and in real time during acquisition. An example long range acquisition image (slant range distance approximately $137 \mathrm{NM}$ ), the image at PCA, the maximum resolution image and the post PCA image showing the Shuttle moving away from Cast Glance are shown in Figs. 16, 17, 18, and 19 respectively. Shown in Figs. 17 and 18 are the successfully imaged ABLT and DTO BLT. The modified tile "speed bump" induced the DTO BLT. The larger ABLT was unexpected and produced by an unknown anomaly near the Shuttle's front landing gear door ${ }^{1}$.

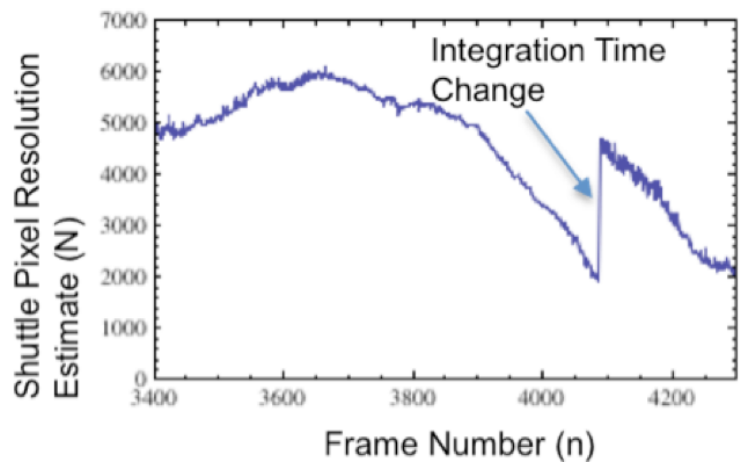

Fig. 14. Estimate of Shuttle pixel resolution.

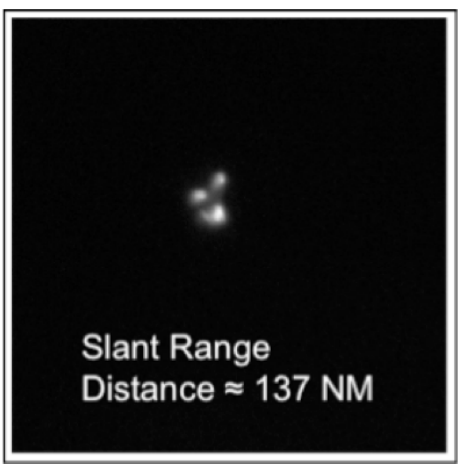

Fig. 16. Long range acquisition.

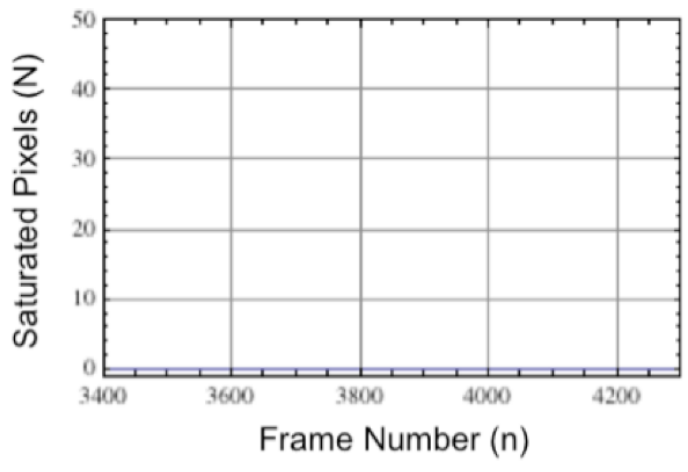

Fig. 15. Number of saturated pixels.

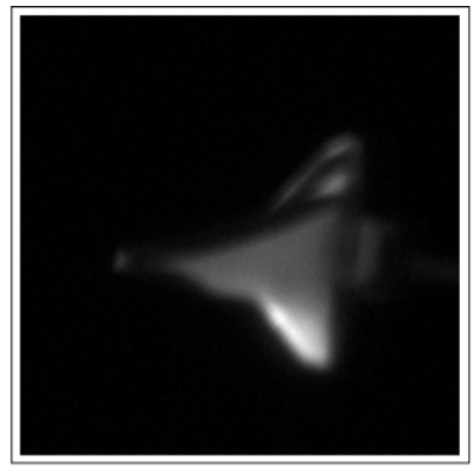

Fig. 17. PCA image. 


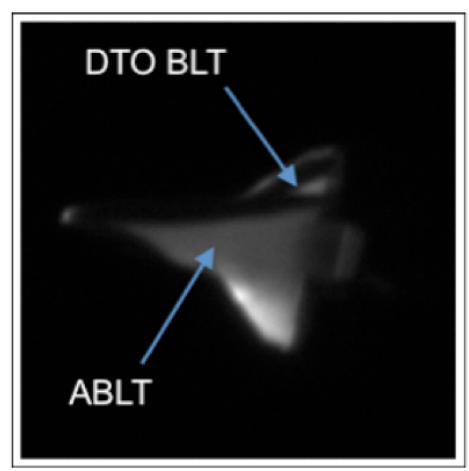

Fig. 18. Peak resolution image.

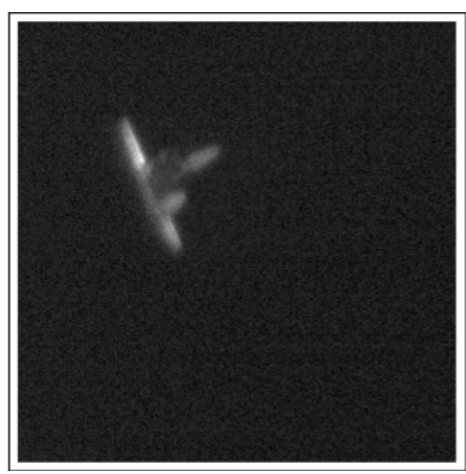

Fig. 19. Post PCA with the Shuttle receding.

\section{B. STS-125 Mission}

Approximately two months later, the same Navy aircraft successfully monitored the surface temperatures of STS-125 Shuttle Atlantis, OV-104, (not configured with a speed bump) during its return from the successful Hubble repair mission. The digital NIR imaging system, captured STS-125 Shuttle mission (May 24, 2009) during re-entry at PCA slant range of approximately $36.5 \mathrm{NM}$ at GMT $=15: 24: 37$, Fig. 20. The total image acquisition time was over 9 minutes, the total number of acquired frames $\mathrm{n}=10,603$ and the integration time was varied from $40 \mathrm{~ms}$ down to $6 \mathrm{~ms}$, Fig. 21. The integration time, for this mission, was rapidly increased as the Shuttle receded to capture potential temperature data on the Shuttle's main engine. The NIR acquisition file size was over 21 Gigabytes. Typically it is expected the best resolution is obtained at PCA. At PCA, image number $=6508$, the pixel resolution is not optimal, but is greatest at image number 6416. This is because the Shuttle is turning away from the aircraft at PCA. This was determined using Eq. (1). Again because the sky background is cool compared to the with the Shuttle's windward surface which is hot (higher pixel counts), the number of pixels associated with the Shuttle can be estimated by summing all the pixels associated with a value greater than 250 counts $(k=250)$. This removes most pixel values associated with the background. This is plotted in Fig. 22 around the PCA frame numbers. The step down, in Fig. 22, is due to the RCS firing shutting down. For STS-125 frame number $=6416$ provided the best pixel resolution, $N=5,476$, with a corresponding slant range distance of $37.5 \mathrm{NM}$ at GMT $=15: 24: 35$. At this point the Shuttle was traveling at approximately Mach 14.3. The image histogram analysis technique determines which image or frame provided the optimal pixel resolution. Fig. 23 shows very few pixels were saturated before the PCA and no saturation around the optimal pixel resolution image. This is due to the ability to adjust the integration time manually, in fine steps and in real time during acquisition. An example long range acquisition image (slant range distance approximately $133 \mathrm{NM}$ ), the image at PCA, the maximum resolution image, RCS thruster firing event and the post PCA image showing the Shuttle moving away from Cast Glance are shown in Figs. 24, 25, 26,27 and 28 respectively. The observed RCS thruster events were useful to verify the timing between the Cast Glance NIR acquisition times and the Shuttle's onboard clock.

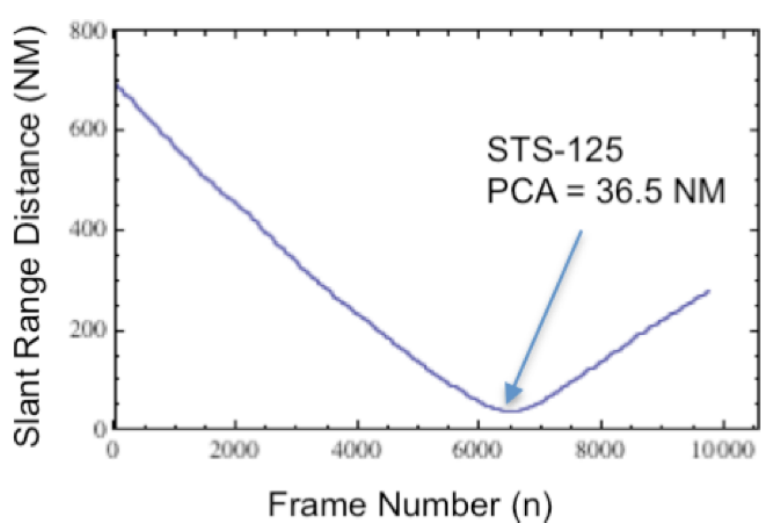

Fig. 20. STS-125 Cast Glance and Shuttle distance.

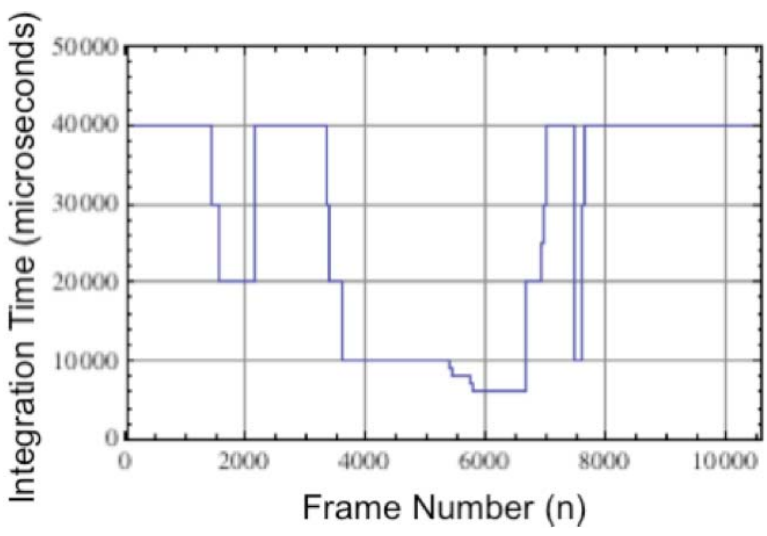

Fig. 21. NIR camera integration times. 


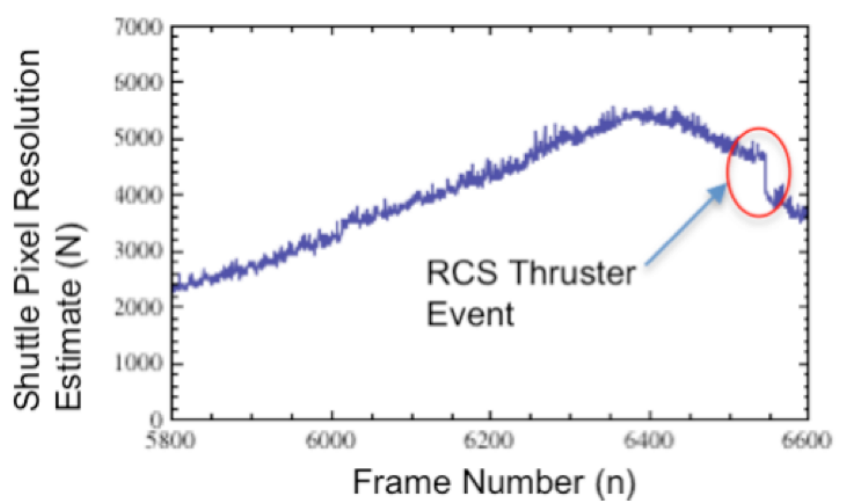

Fig. 22. Estimate of Shuttle pixel resolution.

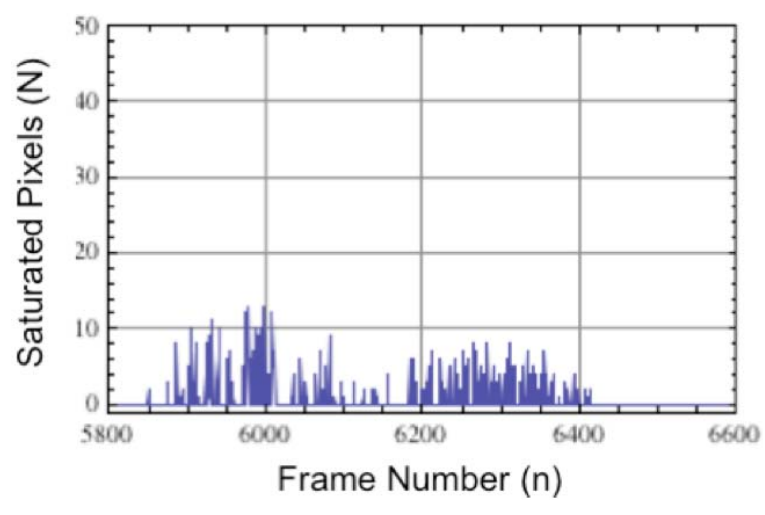

Fig. 23. Number of saturated pixels.

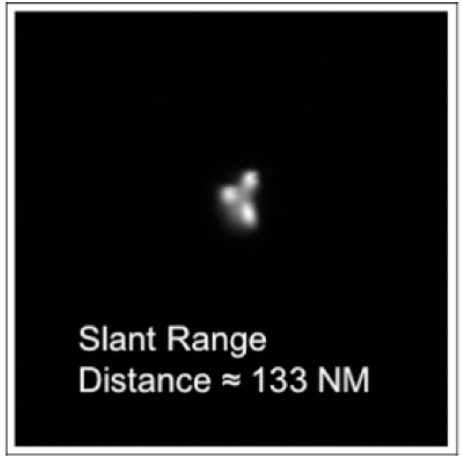

Fig. 24. Long range acquisition.

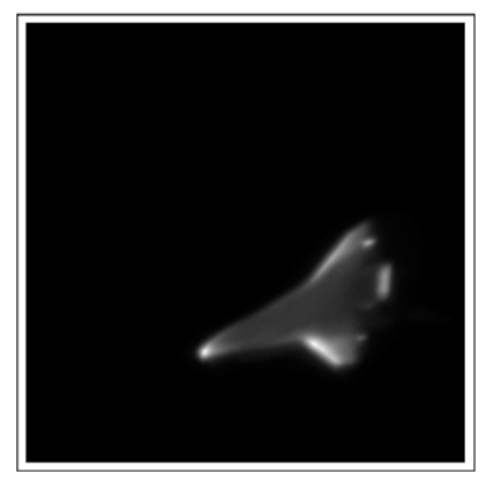

Fig. 25. PCA image.

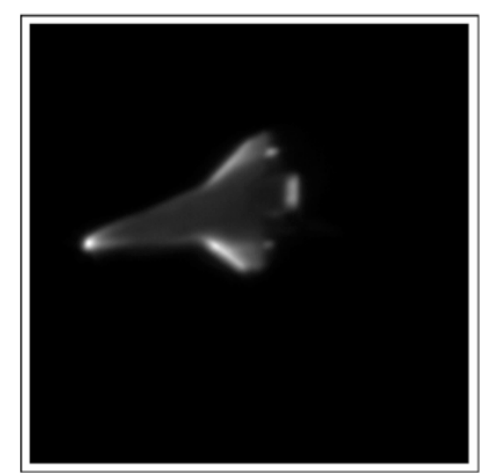

Fig. 26. Peak resolution image.

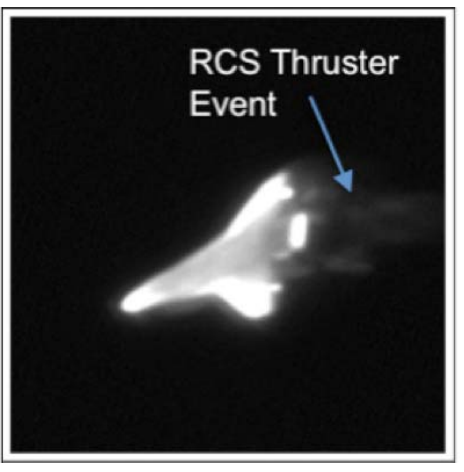

Fig. 27. RCS thruster firing.

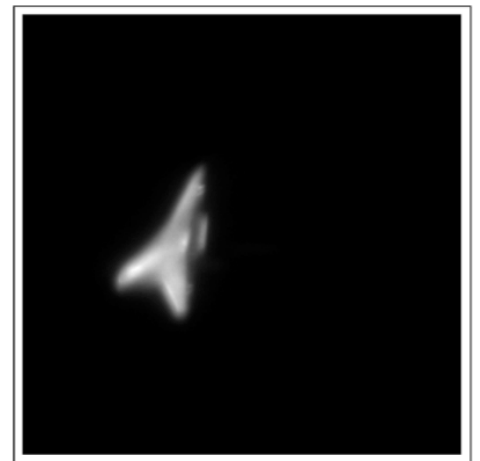

Fig. 28. Post PCA with the Shuttle receding.

\section{STS-128 Mission}

In the fall of 2009 for STS-128 Shuttle Discovery, OV-103, NASA flew a second specially modified thermal protection system tile and instrumentation package to monitor heating effects from BLT during re-entry ${ }^{2}$. This 
speed bump was slightly higher than the speed bump introduced for STS-119. Similar to STS-119, the windward airflow on the port wing was deliberately disrupted to promote transition from laminar flow to turbulence. The digital NIR imaging system, captured STS-128 Shuttle mission (September 12, 2009) during re-entry at a PCA slant range of approximately 41.3 NM as shown in Fig. 29. The total image acquisition time was over 9 minutes, the total number of acquired frames $\mathrm{n}=11,160$ and the integration time was varied from $80 \mathrm{~ms}$ down to $3 \mathrm{~ms}$, Fig. 30 . The NIR acquisition file size was over 21 Gigabytes. At PCA, image number $=6380$, the pixel resolution is not at a maximum at PCA but is at a maximum at image number 6257. This was determined using Eq. (1). The number of pixels associated with the Shuttle was estimated by summing all the pixels associated with a value greater than 100 counts $(k=100)$. This value is lower than previous missions because the background atmosphere was clearer and a relatively lower integration time was used. This is plotted in Fig. 31 around the PCA frame numbers. At image number 6257 and GMT $=00: 38: 46$, the estimated pixel resolution $\mathrm{N}=5399$ at a slant range distance of $43.0 \mathrm{NM}$. At this point the Shuttle was traveling at Mach 14.7. Fig. 32 shows very few pixels were saturated before the PCA and maximum resolution images and no saturation around the maximum resolution image. For some frames the number of pixels saturated was minimal (less than 30). This is again due to the ability to adjust the integration time manually, in fine steps and in real time during acquisition. An example long range acquisition image (slant range distance approximately $130 \mathrm{NM}$ ), the image at PCA, the maximum resolution image, RCS thruster firings, the post PCA image showing the Shuttle moving away from Cast Glance, and contrail image are shown in Figs. 33, 34, 35, 36, 37 and 38 respectively. Figs. 34, 35 and 37 show the DTO BLT imaged successfully.

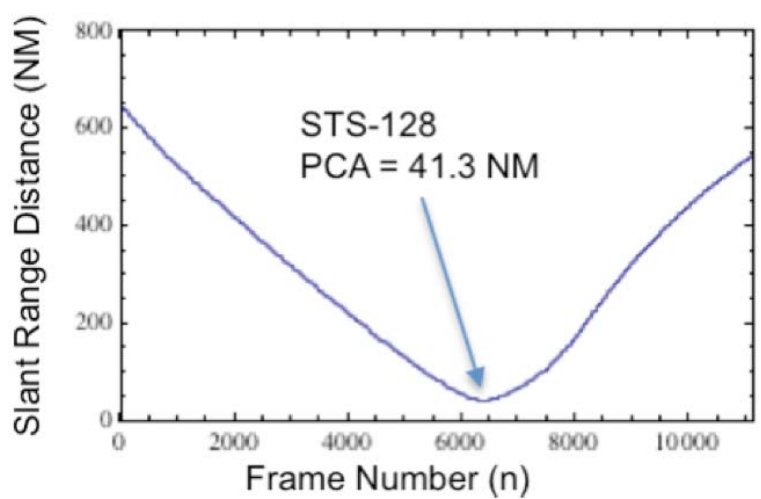

Fig. 29. STS-128 Cast Glance and Shuttle distance.

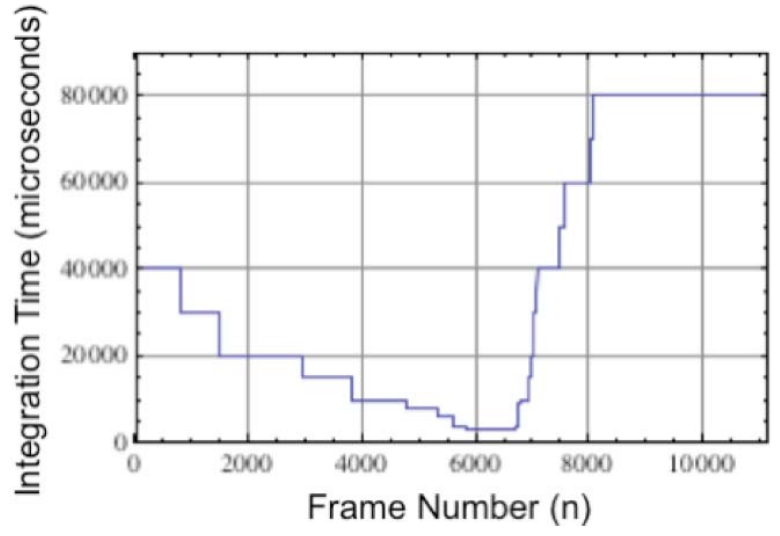

Fig. 30. NIR camera integration times.

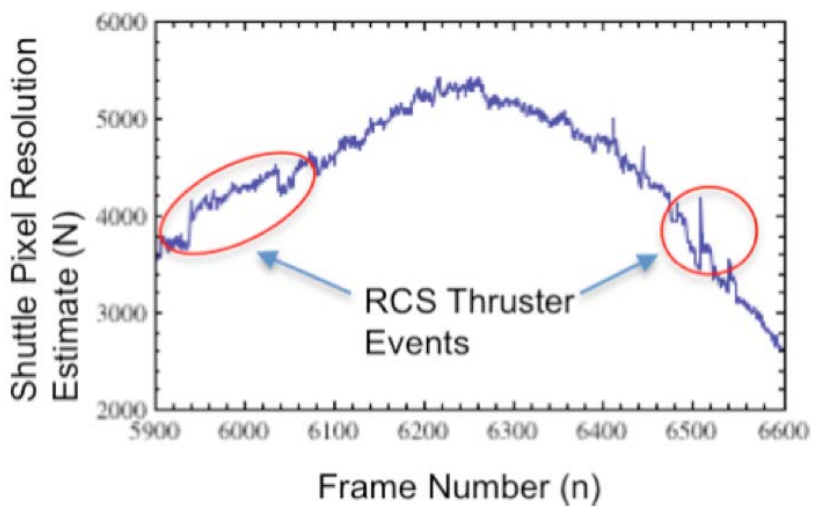

Fig. 31. Estimate of Shuttle pixel resolution.

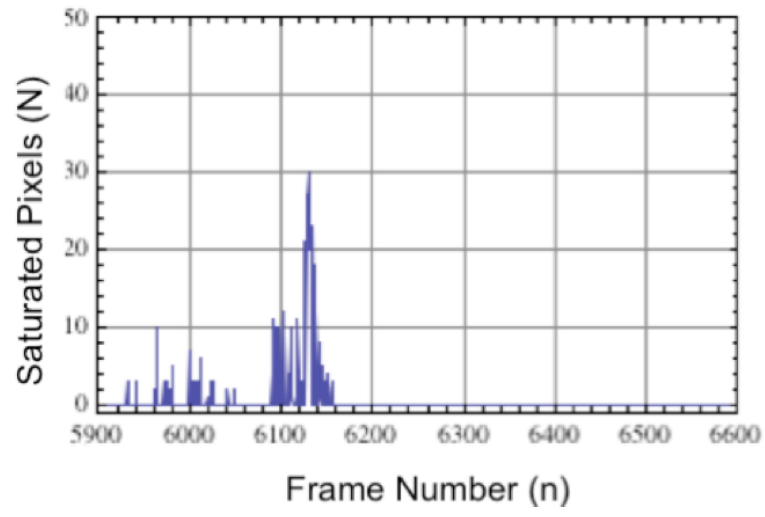

Fig. 32. Number of saturated pixels. 


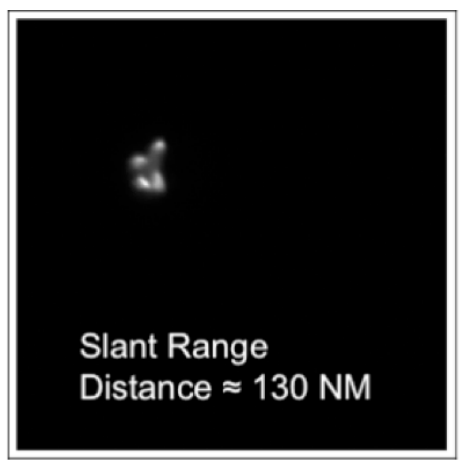

Fig. 33. Long range acquisition.

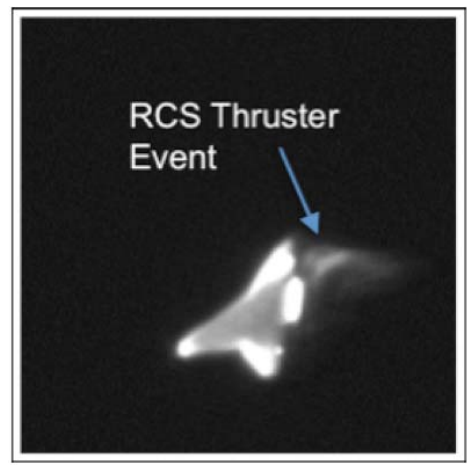

Fig. 36. RCS thruster firing.

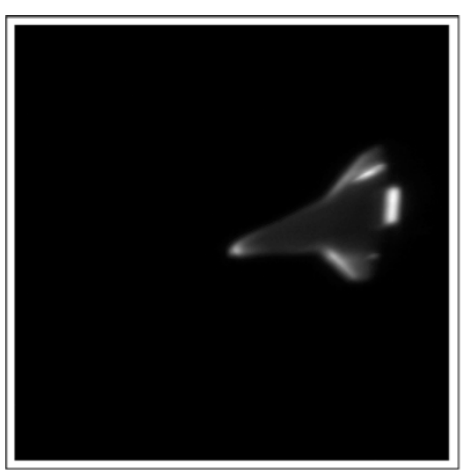

Fig. 34. PCA image.

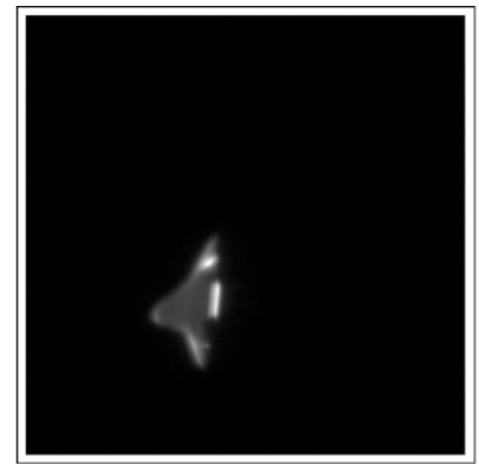

Fig. 37. Post PCA with Shuttle receding.

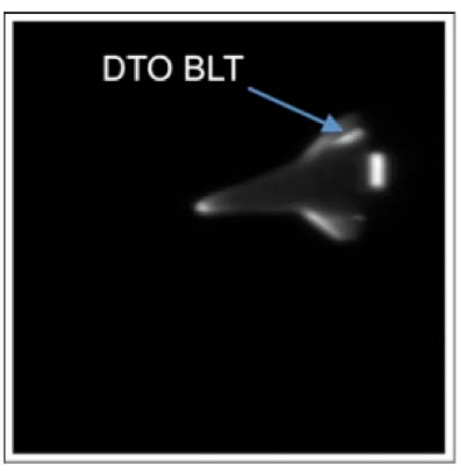

Fig. 35. Peak resolution image.

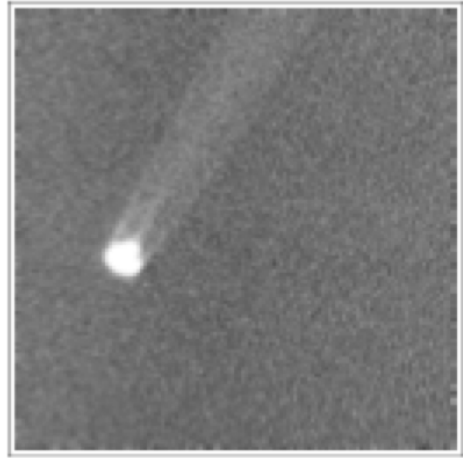

Fig. 38. Contrail image.

\section{Conversion of Acquired Data to 2-Dimensional Temperature Imagery}

Typically after the Shuttle NIR acquisition, there is a quick look analysis to convert the raw intensity counts to a temperature image in a short period of time (several days). The quick look provides temperature imagery for review of significant Shuttle events such as Shuttle anomaly hot spots, BLT areas, and BLT onset and development. The process used to convert the raw image counts to quantitative temperature is shown in Fig. 39. The quick look analysis involves digital image stabilization of the entire sequence of images acquired (around 10,000 - 11,000 frames). The digital stabilization routine uses a frame-to-frame image correlation algorithm. The stabilized sequence is then reviewed for extent of blurring due to atmosphere and optics vibrations, contrail events, RCS firings, etc. The selected sequences of images are then converted to temperature imagery. The calibration curve is used to covert counts to a radiance image for a given integration time. The radiance is corrected for atmospheric path transmission, solar scattering from aerosols, and path radiance to produce the in-band radiance at the Shuttle's windward surface using MODTRAN atmospheric modeling. The object radiance is converted to temperature by using Planck's black body radiation law and the estimated in-band averaged RCG coated tile emissivity value of 0.65 is used ${ }^{11,15}$. The sequence of images providing best resolution is determined using the image histogram analysis technique described previously. Simple boxcar frame averaging can be used on a short sequence of frames ( 8 frames) where the Shuttle orientation, view angle distortions, and optical blurring are minimal. The quick look temperature images for STS119, STS-125, and STS-128 Shuttle missions are shown in Figs. 40a (8 frame average), 41a, and 42a respectively. These quick look images are intended to provide preliminary temperature values. It is expected that more rigorous analysis of the data using 3-dimensional mapping, frame to frame registration with averaging, correcting for the detailed spectral response of the imaging system, and application of spatial filtering using the PSF will produce more accurate spatially resolved temperature images for eventual comparison to CFD models and wind tunnel results. Some of these advanced processing techniques are applied and discussed in Ref. 8. 


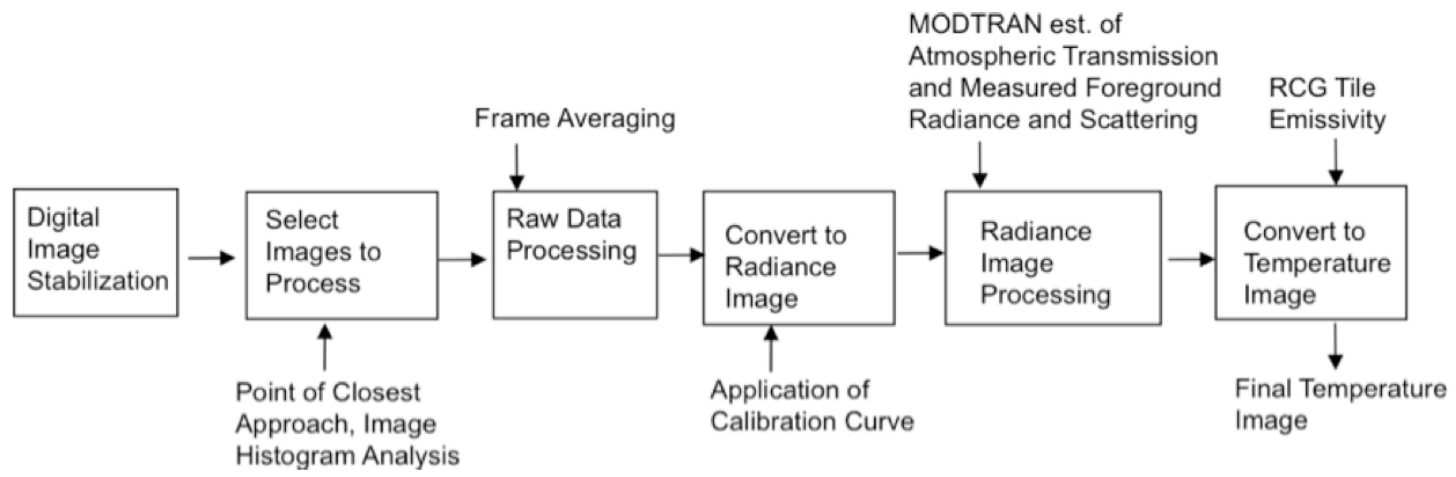

Fig. 39. Conversion of raw intensity imagery to quick look temperature.

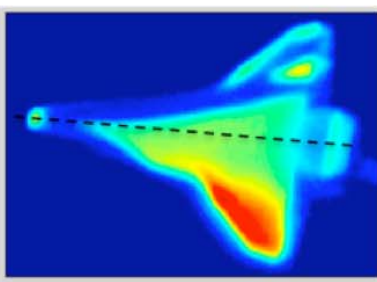

(a)
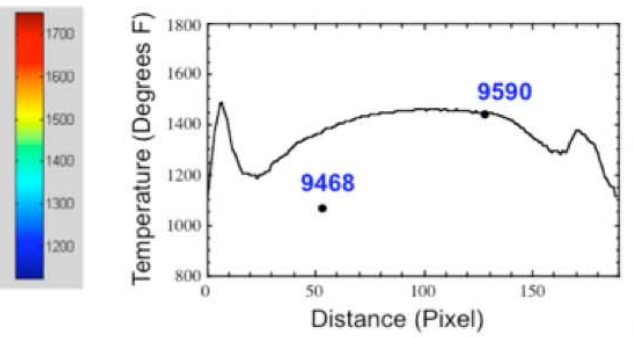

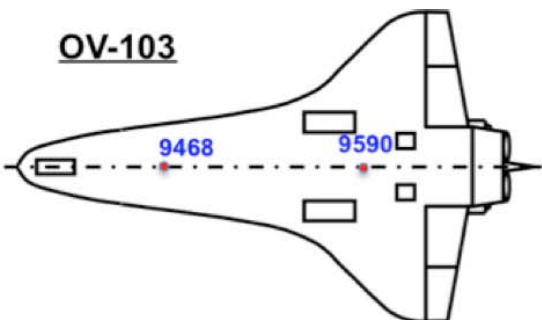

(b)

Fig. 40. STS-119 temperature image with centerline temperature plot and thermocouple comparison.

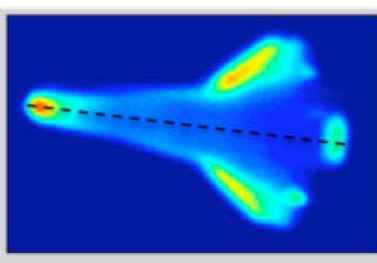

(a)

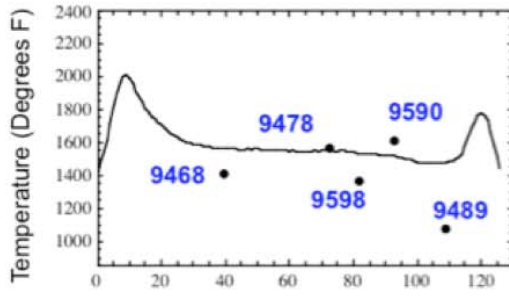

Distance (Pixel)

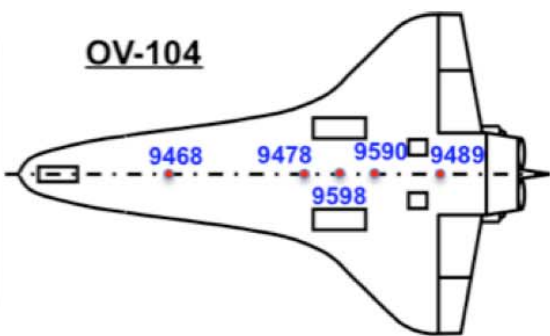

(b)

Fig. 41. STS-125 temperature image with centerline temperature plot and thermocouple comparison.

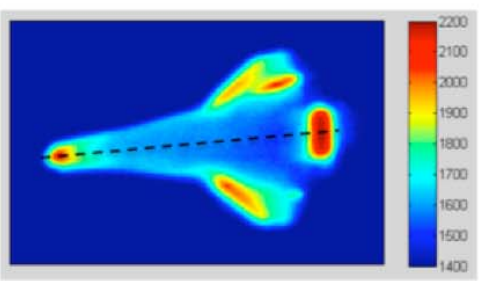

(a)

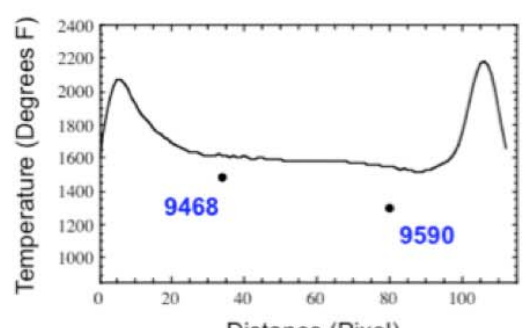

Distance (Pixel)

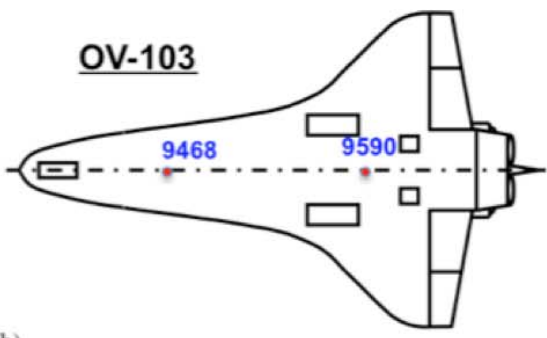

(b)

Fig. 42. STS-128 temperature image with centerline temperature plot and thermocouple comparison. 


\section{Quick Look Temperature Measurements Compared to Thermocouple Values}

The quick look thermal imagery obtained from the HYTHIRM missions is compared to the Shuttle's onboard thermocouples values for STS-119, STS-125, and STS-128 in Figs. 40b, 41b and 42b respectively. The comparisons were performed on the thermocouples located along the centerline. The actual locations of the thermocouples are in 3-dimensional space due to the curvature of the Shuttles windward surface. Using the centerline simplifies location of the thermocouples along 1-dimension. The averaged magnitude difference and averaged ${ }^{\circ} \mathrm{F}$ percent difference error were $172^{\circ} \mathrm{F}$ and 14.1 percent respectively. It is important to note the percent errors are greater when measuring lower temperatures (around $1100^{\circ} \mathrm{F}$ ) due to the spectral limitation of the NIR sensor. Omitting thermocouple temperatures less than $1100^{\circ} \mathrm{F}$ reduces the averaged magnitude difference and averaged ${ }^{\circ} \mathrm{F}$ percent difference error to $119^{\circ} \mathrm{F}$ and 8.5 percent respectively. These values are feasible for the quick look calculations. There are uncertainties associated with the thermocouples that must be quantified for a proper comparison. Typically, the thermocouple offset error is $20^{\circ} \mathrm{F}$ cooler than the actual surface with uncertainties around $+/-20^{\circ} \mathrm{F}^{2}$. However, the OV-104 data seems to exhibit some currently unexplained behavior as seen in Fig. $41 \mathrm{~b}$ where the centerline thermocouple values were scattered with some temperature differences of over $200^{\circ} \mathrm{F}$. This is not expected over a region of laminar flow (temperatures should be uniformly decreasing spatially from nose to aft) and suggests some additional thermocouple offset errors that have not been accounted for. This behavior is currently under investigation. It is expected that a more sophisticated mapping of the 2-dimensional image to the actual 3-dimensional surface of the Shuttle's windward surface will allow for a more accurate determination of the location of the thermocouple in relation to the temperature image and this will help to reduce errors especially in areas of large thermal gradients (note thermocouple \#9468 in Fig. 40b). Also the spectral response of the NIR system was not used to produce the quick look images. The planned determination and application of the detailed spectral response of the imaging system will help to reduce errors. Solar reflection from the RCG coated black tiles is not expected to be significant, but may contribute. The quick look examination of the NIR thermal image data shows low noise within the desired temperature range, while providing global coverage across the orbiter windward surface. It is expected that future efforts ${ }^{8}$ implementing the 2-dimensional to 3-dimensional mapping, applying the spectral response, application of spatial filtering using the PSF, and applying advanced image registration with frame averaging will improve the spatial accuracy of the HYTHIRM generated spatially resolved temperature values with some improvement of the already low noise values. After the additional processing, these temperature values can be used to verify computation models ${ }^{16}$.

\section{Summary}

Implementation of the digital NIR camera system has resulted in NIR imagery with improved dynamic range, instantaneous field of view, and signal to noise. Minimal saturation was obtained for STS-119, STS-125, and STS128 thermal observations. The acquired data were able to image the BLT DTO for both STS-119 and STS-128. In addition an ABLT of unknown origin was imaged for STS-119. Also the quality of the NIR imagery allowed for detailed analysis of the collected data using an image histogram analysis method. The image histogram analysis technique determined which image or frame provided maximum pixels on target based on a combination of PCA and optimal viewing angle (normal incidence to the Shuttle's windward surface) and also was able to detect RCS firings. The quick look temperature images provided good agreement, to within 8.5 percent, of the Shuttle's onboard thermocouples for temperature values above $1,100^{\circ} \mathrm{F}$. It is important to note the errors, both random and systematic, due to the onboard thermocouples needs to be quantified. Lastly, it is expected that implementing the 2dimensional to 3-dimensional mapping, applying the spectral response of the optics, spatial filtering to de-blur the image, and applying advanced image registration with frame averaging will improve the utility of the HYTHIRM generated temperature imagery.

\section{Acknowledgments}

The authors would like to acknowledge the fact that without the assistance of the following organizations and individuals the ambitious work performed under the HYTHIRM project would not have been possible. The authors gratefully acknowledge their contributions and behind-the- scenes work:

- Richard Schwartz, Karen Berger, Scott Splinter, Tom Spisz, Harry Verstynen, Kamran Daryabeigi, Mike Alexander, Scott Berry, Arna Majcher, Andrew McCrea, Paul Krasa, and the rest of the HYTHIRM team, NASA LaRC for perfect mission execution. 
- Dan Hand, CSC/ISTEF for technical support pertaining to calibration and instrumentation hardware/software upgrades.

- $\quad$ Robbie Kerns, LaRC Space Operations Program Office for advocacy and support.

- Jim Hochstetler for calibration logistical support at NAS Corpus Christi, Texas.

- Ron Dantowitz and Marek Kozubal, MARS Personnel, Clay Center Observatory Dexter Southfield Schools.

- Mike Hernandez, Keena Odeghe, and the entire Cast Glance Personnel, Naval Air Warfare Center - Weapons Division US Navy for extreme dedication and ultimately mission execution.

- Stephen Tedford, Commanding Officer, and the entire VX-30 "Bloodhounds" squadron and maintenance personnel for getting HYTHIRM to the right spot in the sky.

- $\quad$ Chuck Campbell and Brian Anderson, NASA JSC for technical consultation and coordination related to the BLT Flight Experiment

- Jennifer Gruber, Mark McDonald and the Flight Dynamics Group, NASA JSC for providing invaluable mission planning support

- John Shannon and LeRoy Cain, JSC Shuttle Program for advocacy and support.

- Tim Orham and the entire Spaceflight Meteorological Group at NASA JSC for their weather forecasting capabilities.

- $\quad$ Nicole Lamotte, Olman Carvajal, Peter Jang, and Susan Kwong, Boeing/USA for decent flight trajectories and consultation pertaining to navigation, aerodynamics and the Shuttle instrumentation database.

- Michael Werner and Don Rudy, The Aerospace Corporation for mission planning and initial radiance model development and application.

- Don Noah, Terri Murphy, Brenda Eliason and Tracy Calhoun, NASA JSC for insight into the Shuttle onorbit imaging process and advocacy.

- Mike Kelly and Kwamee Osei-Wusu, Applied Physics Laboratory for technical expertise and image processing; Jim Kouroupis and John Watson, Applied Physics Laboratory for asset identification and technical consultation.

- Dan Dexter and the entire CEL imaging lab personnel, NASA JSC for graphical-based mission planning.

- Angelo Guastaferro, NIA for project management consultation, leadership and wisdom.

- Richard Wheless, NCI Information Systems and Laura Bass, SAIC for assistance in illustration preparation for proposals, briefings and this manuscript.

- Bob Blanchard, The George Washington University for re-entry imaging inspiration and technical consultation.

- $\quad$ Andrew McCrea, Test Environment Visualization and Support, ATK Space Division, NASA Langley Research Center.

- $\quad$ Bill Wood, NASA LaRC for contributions to radiance modeling and CFD support.

\section{References}

${ }^{1}$ Horvath, T. J., Tomek, D. M., Berger, K. T., Splinter, S. C, Zalameda, J. N., Krasa, P. W., Tack, S., Schwartz, R. J., Gibson, D. M., and Tietjen A. B., "The HYTHIRM Project: Flight Thermography of the Space Shuttle during Hypersonic Reentry," AIAA Paper 2010-241, Jan. 2010.

2 Anderson, B., Campbell, C., Kinder, J., and Saucedo, L., "Boundary Layer Transition Flight Experiment Overview and In-Situ Measurements," AIAA-2010-420, Jan. 2010.

3 Tack, S., Tomek, D. M., Horvath, T. J., Verstynen, H. A., and Shea, E. J., "Cast Glance Near Infrared Imaging Observations of the Space Shuttle during Hypersonic Re-entry," AIAA Paper 2010-243, Jan. 2010.

${ }^{4}$ Horvath, T., Berry, S., Alter, S., Blanchard, R., Schwartz, R., Ross, M., and Tack, S., "Shuttle Entry Imaging Using Infrared Thermography," AIAA-2007-4267, June 2007.

5 Berry, S., Horvath, T., Schwartz, R., Ross, M., Campbell, C., and Anderson, B., "IR Imaging of Boundary Layer Transition Flight Experiments," AIAA-2008-4026, June 2008.

6 Ross, M., Werner, M., Mazuk, S., Blanchard, R., Horvath, T., Berry, S., Wood, W., and Schwartz, R., "Infrared Imagery of the Space Shuttle at Hypersonic Entry Conditions," AIAA-2008-0636, 46th AIAA Aerospace Sciences Meeting and Exhibit, Reno, NV, Jan. 7-10, 2008. 
7 Horvath, T., Berry, S., Splinter, S., Daryabeigi, K., Wood, W., Schwartz, R., and Ross, M., “Assessment and Mission Planning Capability For Quantitative Aerothermodynamics Flight Measurements Using Remote Imaging,” AIAA-2008-4022, June 2008.

8 Gibson, D. M., Spisz, T. S., Taylor, J. C., Zalameda, J. N., Horvath, T. J., Tomek, D. M., Tietjen, A. B., Tack, S., and Bush, B. C., "HYTHIRM Radiance Modeling and Image Analyses in Support of STS-119, STS-125, and STS-128 Space Shuttle Hypersonic Re-entries," AIAA Paper 2010-244, Jan. 2010.

9 A. Berk, L.S. Bernstein, and D.C. Robertson, "MODTRAN: A moderate resolution model for LOWTRAN7", Report GL-TR89-0122, Air Force Geophysics Lab., Bedford, MA, 1989.

${ }^{10}$ Splinter, S., Daryabeigi, K., Horvath, T., Mercer, C.D., Ghanbari, C. M., Ross, M. N., Tietjen, A., B., and Schwartz, R., J., "Solar Tower Experiments for Radiometric Calibration and Validation of Infrared Imaging Assets and Analysis Tools for Entry Aero-Heating Measurements," AIAA-2008-4025, June 2008.

11 Tietjen, A. B. and Hand, D. "Calibration and Data Analysis Summary Report-Hythirm Solar Tower Test," HYTHIRM Internal Report, Sept. 2008.

12 Rybicki G. B. and Lightman, A. P., "Radiative Processes in Astrophysics, New York: John Wiley and Sons, ISBN 0-47182759-2, 1979.

13 Hanisch, R. J., Farris, A., Greisen, E. W., Pence, W. D., Schlesinger, B. M., Teuben, P. J., Thompson, R. W., and Warnock, A., Definition of Flexible Image Transport (FITS), Astronomy \& Astrophysics, 376, 359-380, 2001.

14 Tietjen, A. B. and Hand, D. "Calibration and Data Analysis Summary Report-Hythirm STS-119, STS-125 and STS-128," HYTHIRM Internal Report, Dec. 2009.

15 Bouslog, S. A. and Cunnington, G. R., "Emittance Measurements of RCG Coated Shuttle Tiles", AIAA Paper 92-0851, Jan. 1992.

${ }^{16}$ Wood, W. A., Kleb, W. L., Tang, C. Y., Palmer, G. E., Hyatt, A. J., Wise, A. J., and McCloud, P. L., "Comparison of CFD Predictions with Shuttle Global Flight Thermal Imagery and Discrete Surface Measurements," AIAA Paper 2010-454, Jan. 2010. 